quant-ph/9904009

\title{
New possibilities for supersymmetry breakdown in quantum mechanics and second order irreducible Darboux transformations
}

\author{
Boris F. Samsonov \\ Department of Quantum Field Theory, Tomsk State University, 36 Lenin Ave., \\ 634050 Tomsk, Russia, email: samsonov@phys.tsu.ru
}

\begin{abstract}
.
New types of irreducible second order Darboux transformations for the one dimensional Schrödinger equation are described. The main feature of such transformations is that the transformation functions have the eigenvalues grater then the ground state energy of the initial (or reference) Hamiltonian. When such a transformation is presented as a chain of two first order transformations, an intermediate potential is singular and therefore intermediate Hamiltonian can not be Hermitian while the final potential is regular and the final Hamiltonian is Hermitian. Second derivative supersymmetric quantum mechanical model based on a transformation of this kind exhibits properties inherent to models with exact and broken supersymmetry at once.
\end{abstract}

PACS: 03.65.Ge; 03.65.Fd; 03.65.Ca

Keywords: Supersymmetric quantum mechanics, Darboux transformation, Schrödinger equation, exact solutions

1. Supersymmetric quantum mechanics (SUSY QM) that has been introduced to illustrate problems of supersymmetry breakdown in quantum field theories finds now numerous applications in different fields of theoretical and mathematical physics (for a review see [1]). It is well known that the supersymmetry may be either exact or broken. In the case of the broken supersymmetry the entire spectrum of the supehamiltonian is degenerate while in the case of the exact one its vacuum state is nondegenerate. We would like to stress that other possibilities exist as well [2, 3] that may have applications in quantum field theories [2].

In the conventional SUSY QM [1] supercharges are built of first order Darboux transformation operators 4 . Higher order Darboux transformation operators are involved in a higher derivative supersymmetry [5, 6]. In the simplest case we have a second order derivative supersymmetry with the supercharges built of the second order Darboux transformation operators. It is known that such a supersymmetry may be either reducible or not [7]. The concept of complete reducibility is introduced as well [3, 8]. This concept is based on a theorem that establishes the equivalence between an $N$-order Darboux transformation and a chain of $N$ first order Darboux transformations [8].

Every chain of $N$ first order Darboux transformations creates a chain of exactly soluble Hamiltonians $h_{0} \rightarrow h_{1} \rightarrow \ldots \rightarrow h_{N}$. We suppose that $h_{0}$ and $h_{N}$ are Hermitian in a Hilbert space and admit unique self-adjoint extensions (i.e. they are essentially self-adjoint). To satisfy this condition the potentials $V_{i}(x), h_{i}=-\partial_{x}^{2}+V_{i}(x), \partial_{x}^{2}=\partial_{x} \partial_{x}, \partial_{x}=d / d x, i=0, N$ should be realvalued and free of singularities in their common domain of definition $(a, b)$ where $a$ or $b$ or both may be equal to $+\infty$ or $-\infty$. Any chain is called reducible [7] if all intermediate potentials $V_{1}(x), \ldots, V_{N-1}(x)$ are realvalued functions defined in $(a, b)$. The 
$N$ th order Darboux transformation which is equivalent to the whole chain is called reducible as well. When at least one of the intermediate potentials is a complexvalued function the chain and the corresponding $N$ th order transformation are called irreducible. Some reducible chain is called completely reducible [2] if all these potentials are free of singularities in $(a, b)$. The corresponding $N$ th order transformation is called completely reducible as well.

2. It can be shown that every $N$ th order Darboux transformation is equivalent to the resulting action of a chain of well-defined transformations of order less or equal two. There is a marvellous vast literature devoted to the analysis of first order transformations (see e.g. [1]). Second order transformations are not explored in the same details. The main purpose of this letter is to fulfil this gap and give an analysis of second order transformations.

Let $L$ be a second order Darboux transformation operator defined as an operator that intertwines two well-defined Hamiltonians $h_{0}$ and $h_{2}, L h_{0}=h_{2} L$. Every operator of this type may be presented in a compact form as follows [8]: $L \psi=W^{-1}\left(u_{1}, u_{2}\right) W\left(u_{1}, u_{2}, \psi\right)$ where $W$ stands for the usual symbol of a Wronskian and the functions $u_{1}(x)$ and $u_{2}(x)$ called transformation functions are eigenfunctions of $h_{0}, h_{0} u_{1,2}=\alpha_{1,2} u_{1,2}$ which are not supposed to satisfy any boundary conditions. When supercharge operators $Q$ and $Q^{+}$are built in terms of $L$ and $L^{+}$where $L^{+}$is Laplace adjoint to $L$

$$
Q=\left(\begin{array}{cc}
0 & L^{+} \\
0 & 0
\end{array}\right), \quad Q^{+}=\left(\begin{array}{cc}
0 & 0 \\
L & 0
\end{array}\right)
$$

then these operators together with the superhamiltonian $\mathcal{H}=\operatorname{diag}\left(h_{0}, h_{2}\right)$ close a second order superalgebra: $Q^{2}=\left(Q^{+}\right)^{2}=0, Q Q^{+}+Q^{+} Q=\left(\mathcal{H}-\alpha_{1} \mathcal{I}\right)\left(\mathcal{H}-\alpha_{2} \mathcal{I}\right)$ where $\mathcal{I}$ is the unit $2 \times 2$ matrix. The new potential $V_{2}(x)$ is defined by the initial potential $V_{0}(x)$ and the transformation functions $V_{2}(x)=V_{0}(x)-2\left[\log W\left(u_{1}, u_{2}\right)\right]^{\prime \prime}$ where the prime stands for the derivative with respect to $x$.

The operator $L$ can always be presented as a superposition of first order operators $L=$ $L^{(1)} L^{(2)}$ where $L^{(1)}=-\partial_{x}+\left(\log u_{1}\right)^{\prime}, L^{(2)}=-\partial_{x}+(\log v)^{\prime}, v=L^{(1)} u_{2}$. The intermediate potential of such a chain of transformations is defined by the function $u_{1}: V_{1}(x)=V_{0}(x)-$ $2\left(\log u_{1}\right)^{\prime \prime}$.

In the conventional SUSY QM [1] transformation functions are supposed to be such that their eigenvalues are subject to the condition $\alpha_{1,2} \leq E_{0}$ where $E_{0}$ is the ground state energy when $h_{0}$ has a discrete spectrum and it is the low bound of the continuum spectrum when the discrete spectrum is absent. They also may be chosen such that $\alpha_{1}=E_{0}, \alpha_{2}=E_{1}$ where $E_{1}$ is the energy of the first excited state. These choices correspond to the usual conception of the supersymmetry breakdown in quantum mechanics when the vacuum state of $\mathcal{H}$ is nondegenerate for the exact supersymmetry and it is two fold degenerate for the broken supersymmetry. In the latter case the whole spectrum of $\mathcal{H}$ is two fold degenerate.

3. In what follows we shall denote by $E_{k}$ and $\psi_{k} k=0,1, \ldots$ the discrete spectrum eigenvalues and eigenfunctions of the Hamiltonian $h_{0}$ respectively. We will suppose for simplicity that the whole spectrum of $h_{0}$ is discrete.

We shall prove below that $\alpha_{1,2}$ may be chosen such that $E_{k+1} \geq \alpha_{2}>\alpha_{1} \geq E_{k}$. In this case the transformation functions $u_{1,2}$ has nodes in $(a, b)$ and consequently intermediate potential $V_{1}(x)$ has singularities in $(a, b)$. Nevertheless there exists such a choise of the transformation functions that the final potential $V_{2}(x)$ is free of singularities. We obtain thus a simplest irreducible chain of Darboux transformations. 
Such a transformation either deletes one or two energy levels ( $E_{k}$ or $E_{k+1}$ or both) or creates one or two new energy levels disposed between $E_{k}$ and $E_{k+1}$ ( $\alpha_{1}$ or $\alpha_{2}$ or both). The SUSY QM based on this transformation has the properties of the theories with the exact and broken supersymmetry at once. The ground state of $\mathcal{H}$ is two fold degenerate and in the middle of the spectrum of $\mathcal{H}$ there exist one or two nondegenerate energy levels.

4. Let us establish now conditions for the potential $V_{2}(x)$ to be free of singularities in the interval $(a, b)$. For this purpose it is sufficiently to analyse the second order Wronskian $W\left(u_{1}, u_{2}\right)$ as a function of $x$ and find the conditions when it is free of zeros in $(a, b)$.

For the sake of definiteness we will consider the full real axis $\mathbb{R}=(-\infty, \infty)$ as the interval $(a, b)$ and suppose the potential $V_{0}(x)$ to be confining, i.e. $\left|V_{0}(x)\right| \rightarrow \infty$ as $|x| \rightarrow \infty$. We also assume the potential $V_{0}(x)$ to be sufficiently smooth function in $\mathbb{R}\left(\right.$ e.g. $\left.V_{0}(x) \in C_{\mathbb{R}}^{\infty}\right)$ and bounded from below. In this case the operator $h_{0}=-\partial_{x}^{2}+V_{0}(x)$ initially defined on a set of infinitely differentiable functions with a compact support which is dense in the Hilbert space of functions defined over $\mathbb{R}$ and square integrable with respect to the Lebesgue measure has a self adjoint closure that we shall denote $h_{0}$ as well. Moreover, $h_{0}$ has only a discrete spectrum $E=E_{k}$ with the eigenfunctions $\psi=\psi_{k}, k=0,1, \ldots$

It is not difficult to see that every eigenfunction of $h_{0}, \psi_{E}$, with $E$ such that $E_{k+1}>E>$ $E_{k}, k=0,1, \ldots$ may have on $\mathbb{R}$ either $k+1$ nodes or $k+2$ ones. Moreover, If $\psi_{E}$ has $k+2$ nodes then $\left|\psi_{E}(x)\right| \rightarrow \infty$ as $|x| \rightarrow \infty$. If $\psi_{E}$ has $k+1$ nodes then we have two possibilities: a) $\psi_{E} \rightarrow 0$ as $x \rightarrow \infty$ (or equivalently as $x \rightarrow-\infty$ ) and b) $\left|\psi_{E}\right| \rightarrow \infty$ as $|x| \rightarrow \infty$. In the first case we refer $\psi_{E}$ as the function with a zero asymptotic at the right infinity (or equivalently at the left infinity) and in the second case as the one with a growing asymptotic at both infinities. If $\psi_{E}$ is a zero asymptotic function then it has just $k+1$ nodes in $\mathbb{R}$. These assertions are direct implications of the well-known Sturm oscillator theorem (see e. g. 99).

Our analysis shows that if the transformation functions $u_{1}$ and $u_{2}, h_{0} u_{1,2}=\alpha_{1,2} u_{1,2}$ are chosen such that $E_{k+1} \geq \alpha_{2}>\alpha_{1}>E_{k}, k=0,1, \ldots$ and $u_{1}(x)$ has $k+2$ nodes, $u_{2}(x)$ has $k+1$ nodes on $\mathbb{R}$ then $W\left(u_{1}, u_{2}\right)=W(x)$ is free of zeros on $\mathbb{R}$. Really. We note first of all that because of the conditions imposed on $u_{1}$ and $u_{2}$ they have simple and alternating zeros i.e. between any two consecutive zeros of one of them there is exactly one zero of the other. The total number of nodes of the functions $u_{1}$ and $u_{2}$ is odd and equal $2 \mathrm{k}+3$. Let $x_{0}, \ldots, x_{2 k+2}$ be the zeros of the functions $u_{1}(x)$ and $u_{2}(x)$. Since $W^{\prime}(x)=\left(\alpha_{1}-\alpha_{2}\right) u_{1}(x) u_{2}(x)$ where the use of the Schrödinger equation is made the points $x_{0}, \ldots, x_{2 k+2}$ are the points of local minima and maxima of $W(x)$. It then follows that this function is monotone in every interval $\left[x_{j}, x_{j+1}\right], j=0,1, \ldots$ and the points $x_{0}$ and $x_{2 k+2}$ are both either maxima or minima. It is not difficult to see that the sign of the function $W(x)=u_{1}(x) u_{2}^{\prime}(x)-u_{2}(x) u_{1}^{\prime}(x)$ is the same for all $x=x_{0}, \ldots x_{2 k+2}$ and $W(x) \neq 0 \forall x \in\left[x_{0}, x_{2 k+2}\right]$. It remains now to analyse the behaviour of the function $W(x)$ for $x<x_{0}$ and $x>x_{2 k+2}$.

The sign of the functions $u_{1}(x)$ and $u_{2}(x)$ is without importance. Therefor without loss of generality we can always choose $u_{1}(x)$ and $u_{2}(x)$ such that $u_{1,2}(x) \geq 0$ for $x>x_{2 k+2}$. In this case $u_{1}\left(x_{2 k+2}\right)=0, u_{2}\left(x_{2 k+2}\right)>0, u_{1}^{\prime}\left(x_{2 k+2}\right)>0$. We conclude that $W\left(x_{2 k+2}\right)<0$ and $W^{\prime \prime}\left(x_{2 k+2}\right)<0$. This means that $x_{0}$ and $x_{2 k+2}$ are the points of local maxima of $W(x)$. Taking into account the fact that $W(x)$ is monotone for $x>x_{2 k+2}$ and $x<x_{0}$ (since $W^{\prime}(x) \neq 0$ for all these $x$ ) and negative we find that $W(x) \neq 0$ for all $x \in \mathbb{R}$.

5. It is clear from the above considerations that if $u_{1}(x)$ has $k+1$ nodes and $u_{2}(x)$ has $k+2$ nodes then under the same assumptions that above the function $W(x)$ is positive and 
riches the local maxima at $x=x_{0}$ and $x=x_{2 k+2}$. Further, the function $u_{1}(x)$ is not square integrable on $\mathbb{R}$ because of the condition $E_{k+1} \geq \alpha_{2}>\alpha_{1}>E_{k}$. The function $u_{2}(x)$ is not square integrable for all $\alpha_{2} \in\left[E_{k+1}, E_{k}\right)$ since it is assumed to have $k+2$ nodes. It follows then that $\left|u_{2}(x)\right| \rightarrow \infty$ as $|x| \rightarrow \infty$ and $\left|u_{1}(x)\right|$ tends to infinity in one of the infinities at least. This means that $|W(x)|$ tends to infinity in one of the infinities at least and $|W(x)|$ has one node at least.

We could avoid such a behaviour of $W(x)$ if $|W(x)|$ would decrease as $|x| \rightarrow \infty$ instead of increase. For such a behaviour of $|W(x)|$ the function $u_{1}(x)$ should be square integrable over $\mathbb{R}$. (Note that the case when $u_{2}(x)$ is square integrable was considered above.) I could not prove that for an arbitrary potential $V_{0}(x)$ the Wronskian $W(x)$ tends in this case to zero as $|x| \rightarrow \infty$. Nevertheless, I can indicate a wide class of potentials for which the condition $W(x) \rightarrow 0$ as $|x| \rightarrow \infty$ holds. In particularly, all potentials satisfying the condition

$$
\int_{-\infty}^{+\infty}\left|x V_{0}(x)\right| d x<\infty
$$

(scattering potentials) are of this type. As to confining potentials it can be proven that when the potential $V_{0}(x)$ is such that

$$
\int_{-\infty}^{\infty}\left|\frac{V_{0}^{\prime}}{V_{0}^{5 / 4}}\right|^{2} d x<\infty, \quad \int_{-\infty}^{\infty} \frac{\left|V_{0}^{\prime \prime}\right|}{\left|V_{0}\right|^{3 / 2}} d x<\infty .
$$

then $W(x) \rightarrow 0$ as $|x| \rightarrow \infty$. The proof of this assertion is based on the asymptotic behaviour of the solutions of the Schrödinger equation for such potentials which is known (see e.g. [9]) and it is omitted here. This imply another possibilities for the choice of the transformation functions. If they are chosen such that $E_{k+1} \geq \alpha_{2}>\alpha_{1}=E_{k}, u_{1}(x)=\psi_{k}(x)$, and $u_{2}(x)$ has $k+1$ nodes then $W\left(u_{1}, u_{2}\right) \neq 0 \forall x \in \mathbb{R}$. The proof can easily been obtained with the help of the asymptotic behaviour of $u_{1}(x)$ and $u_{2}(x)$.

6. We have formulated conditions that are sufficient to impose on the transformation functions to obtain a regular potential of the transformed Schrödinger equation with the potential $V_{2}(x)$. It can be shown with the aid of the known asymptotic of the solutions of the initial Schrödinger equation that $V_{2}(x) \rightarrow V_{0}(x)$ as $|x| \rightarrow \infty$. Moreover, the knowledge of this asymptotic allows us to find all solutions of the transformed Schrödinger equation that belong to the Hilbert space $L_{2}(\mathbb{R})$. This analysis is based on the following affirmations.

The spectrum of the Hamiltonian $h_{N}$ related with $h_{0}$ by the $N$ th order Darboux transformation coincides with the spectrum of $h_{0}$ with the possible exception of a finite number of discrete levels defined by the choice of the transformation functions. The level $E=\alpha$ is absent in the spectrum of $h_{N}$ if and only if $u_{\alpha} \in \operatorname{Ker} L, h_{0} u_{\alpha}=\alpha u_{\alpha}$ and $u_{\alpha} \in L_{2}(\mathbb{R})$. The level $E=\alpha$ is created in the spectrum of $h_{N}$ if and only if $v_{\alpha} \in \operatorname{Ker} L^{+}, h_{N} v_{\alpha}=\alpha v_{\alpha}$ and $v_{\alpha} \in L_{2}(\mathbb{R})$. The space $\operatorname{Ker} L^{+}$has the basis [8] $v_{1}(x), \ldots, v_{N}(x), v_{j}=W^{(j)}\left(u_{1}, \ldots u_{N}\right) / W\left(u_{1}, \ldots u_{N}\right)$, $h_{N} v_{j}=\alpha_{j} v_{j}$ where $W^{(j)}\left(u_{1}, \ldots u_{N}\right)$ is the Wronskian of order $N-1$ built of the functions $u_{1}, \ldots, u_{N}$ except for the function $u_{j}$.

The direct implication of this assertion is that the functions $\varphi_{k}=L \psi_{k}$ are square integrable over $\mathbb{R}$ for all $\psi_{k} \neq u_{j}$. To find all square integrable solutions of the transformed Schrödinger equation it remains now to analyse the functions $v_{1,2}=u_{2,1} / W\left(u_{1}, u_{2}\right) \in \operatorname{Ker} L^{+}$. This analysis is possible because of the known asymptotic of the solutions of the initial Schrödinger equation. We have obtained the following results. 
If the transformation functions $u_{1,2}$ are such that $E_{k+1}>\alpha_{2}>\alpha_{1}>E_{k}$ and have growing asymptotic on both infinities then $v_{1,2} \in L_{2}(\mathbb{R})$ and the set $\left\{v_{1}, v_{2}, \varphi_{n}=L \psi_{n}, n=0,1, \ldots\right\}$ is complete in $L_{2}(\mathbb{R})$. (Hamiltonian $h_{2}$ has two additional energy levels $E=\alpha_{1}, \alpha_{2}$ with respect to $h_{0}$.) If the function $u_{2}$ has zero asymptotic then $v_{2} \notin L_{2}(\mathbb{R}), h_{2}$ has a single additional energy level $E=\alpha_{1}$, and the set $\left\{v_{1}, \varphi_{n}=L \psi_{n}, n=0,1, \ldots\right\}$ forms a basis in $L_{2}(\mathbb{R})$.

If $\alpha_{2}=E_{k+1}, u_{2}=\psi_{k+1}$, and $u_{1}$ has a growing asymptotic at both infinities then the level $E=E_{k+1}$ is absent in the spectrum of $h_{2}$ and the level $E=\alpha_{1}$ is created. The set $\left\{v_{1}, \varphi_{n}=L \psi_{n}, n=0,1, \ldots ; n \neq k+1\right\}$ is complete in $L_{2}(\mathbb{R})$.

If $\alpha_{1}=E_{k}, u_{1}=\psi_{k}$, and $u_{2}$ has a growing asymptotic at both infinities then the level $E=E_{k}$ is absent in the spectrum of $h_{2}$ and the level $E=\alpha_{2}$ is created. The basis in $L_{2}(\mathbb{R})$ is formed by the set $\left\{v_{2}, \varphi_{n}=L \psi_{n}, n=0,1, \ldots ; n \neq k\right\}$. When $u_{2}$ has a zero asymptotic then we have only a deletion of the level $E=E_{k}$ and the basis is formed by the set $\left\{\varphi_{n}=L \psi_{n}, n=0,1, \ldots ; n \neq k\right\}$. Finally, if $\alpha_{2}=E_{k+1}, \alpha_{1}=E_{k}$, and $u_{1}=\psi_{k}, u_{2}=\psi_{k+1}$ then both levels $E=E_{k}$ and $E=E_{k+1}$ are absent in the spectrum of $h_{2}$ and the position on the other levels is unchanged. The set $\left\{\varphi_{n}=L \psi_{n}, n=0,1, \ldots ; n \neq k, k+1\right\}$ is complete in $L_{2}(\mathbb{R})$. This possibility has been earlier indicated by Krein [10.

7. As a final remark we note that the possibility to use the transformation functions with the eigenvalues higher then the ground state energy of $h_{0}$ has recently been noted in [11] without any analysis.

The financial support from the RFBR and the ministry of education of Russia is gratefully acknowledged.

\section{References}

[1] G. Junker, Supersymmetric methods in quantum and statistical physics. Springer, Berlin, 1996; F. Cooper, A. Khare, U. Sukhatme, Phys. Rep. 251 (1995) 267.

[2] B. Sathiapalan, Phys. Rev. Lett. 55 (1985) 669.

[3] B.F. Samsonov, Mod. Phys. Lett. A. 11 (1996) 1563.

[4] A.A. Andrianov, N.V. Borisov, M.V. Ioffe, Phys. Lett. A 105 (1984) 19.

[5] A.A. Andrianov, M.V. Ioffe, V. Spiridonov, Phys. Lett. A 174 (1993) 273.

[6] V.G. Bagrov, B.F. Samsonov, Theor. Math. Phys. 104 (1995) 356.

[7] A.A. Andrianov, M.V. Ioffe, D.N. Nishnianidze, Theor. Math. Phys. 104 (1995) 463.

[8] V.G. Bagrov, B.F. Samsonov Phys. Part. Nucl. 28 (1997) 374.

[9] F.A. Berezin, M.A. Shubin, The Schrödinger Equation, Moscow University Press, 1983.

[10] M.G. Krein, DAN SSSR 113 (1957), 970.

[11] D.J. Fernandez C., V. Hussin, B. Mielnik, Phys. Lett. A 244 (1998) 309. 